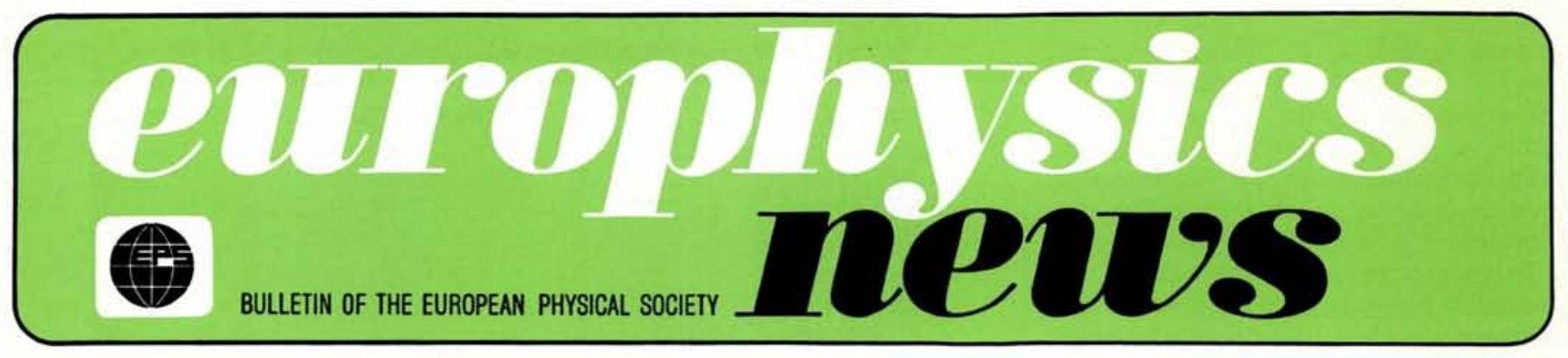

J.A. Volume $9 \quad$ Number $7 / 8$

July/A.ugust 1978

\section{General Conference in York, UK}

Bulletin No. 2 is available on request from the Secretariats of the EPS and I.o.P. Participants are recommended to register immediately.

Physics is held in the highest esteem by Polish society. It has a long-standing tradition and is widely practised. Of the 45 institutes employing some 3500 physicists, the majority have been set up within the framework of university organizations, so that apart from research work the physicists employed there are heavily involved in teaching. Nevertheless, more Polish physicists are employed outside the scientific and higher education organizations. In the primary and secondary schools alone, there are about 5000 teachers who are graduates in physics. The total number of people teaching physics in these schools is, moreover, almost five times greater.

Physics research is conducted in Poland on a broad front, its scope encompassing all the major branches of this discipline. Considerable freedom of choice is left to the researchers themselves, although the general trends are polarized towards the needs of the national economy. A few years ago (1971-1973) a new system of planning and coordination in $\mathrm{R}$ and D was introduced for the whole of science. This system aims to accelerate the integration of science, technology and the national economy, and favours research connected with the modernization of the technologies of particular significance for the further development of the country. In fundamental research, particular stress is laid on those fields of physics in which Polish science has achieved world recognition, and where further contributions of Polish scientists may be expected to be significant.

\title{
Physics in Poland
}

\section{Z. Wilhelmi, Warsaw}

(President of the Polish Physical Society)

In the framework of the national plan for the development of science and technology, a complex system of "crucial ", "interdepartmental" and " intradepartmental" projects has been established.

High priority is given to the 67 crucial projects, which are set down by the Ministry of Science, Higher Education and Technology. Several of them include physics research, both fundamental and applied. For example, the major part of the topics related to nuclear physics are included in the vast problem entitled "Investigation of nuclear processes and utilization of nuclear techniques for the further development of the national economy". The size of a typical crucial project can be gauged from the fact that about 1500 research workers in 40 scientific institutions are engaged in the tasks related to the project: "Physico-chemical Foundations of Technological Processes". Research work conducted within the framework of crucial projects is supported primarily by the national budget and by the so-called "State Research Fund ".

Intradepartmental projects, of which there are in the whole country about 500 , are set down and co-ordinated by the respective governmental departments. They get financial support from the "Technology and Trade Development Fund " which is raised by the industrial unions and combines. Physics research is included, however, in only a few of these projects.

The overwhelming majority of physics investigations, especially in the fundamental field, are conducted through " interdepartmental projects". Even so, among the 58 projects of this type, only 6 are directly physics, viz. :

1) Structure and electronic properties of solids

2) Interaction of radiation with matter

3) Fields, particles and space-time continuum

4) Galaxy, stars and the solar system

5) Structure, theory and properties of molecular and condensed systems

6) Interplanetary and circumterrestrial space.

Such research projects are co-ordinated nation-wide by the Polish Academy of Sciences, which also acts as consultant for the utilization of the scientific findings in the national economy.

Seven R and D problems of particular significance for the country's development have been selected, raised to

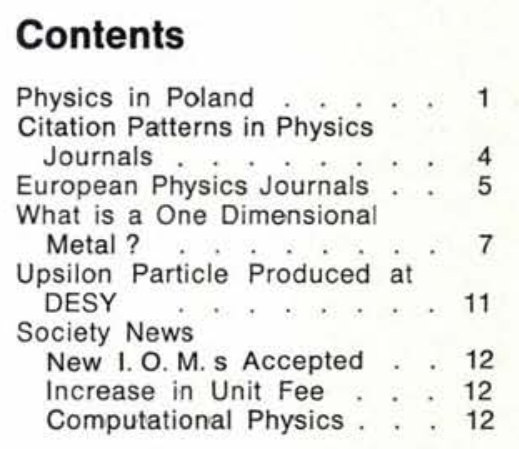

Europhysics News is published monthly by the European Physical Society. (c) 1978. Reproduction rights reserved. 
the standing of the so-called "Governmental R and D programmes" and given the highest priority. One of these projects - Development of Materials and Components for the Electronization of Industry - relates largely to physics.

There are two principal sources of research finance: budget grants and subventions from trade organizations. Total outlay on research in 1977 amounted to $36.10^{\circ}$ zloty, (say $2.10^{9}$ Sw. Fr.) out of which $16.10^{\circ}$ came as budget grants. The outlay corresponds to about $2.0 \%$ of the national income. A considerable part, about $30 \%$, has been spent on the realization of the governmental and crucial projects, whereas about 5.10 zloty, i.e. about $14 \%$ of the total expenditures on $R$ and $D$, are allocated each year to fundamental research with prospective significance for the national economy.

The Polish Academy of Sciences allocates about $65 \%$ of the funds granted for fundamental research to projects related to the exact sciences and technology, physics research benefitting relatively well. It deserves mention that a typical subsidy allocated to a research project in physics amounts, on the average, to $0.3-0.6$ $\times 10^{6}$ zloty per researcher per year. Research work conducted in non-academic institutes is usually subsidized more than that conducted at universities, where the workers engaged also in teaching, are financed from other sources. It should be noted that the above figures do not include expenditures on large investments such as laboratory buildings, accelerators, etc.

The distribution of research effort between the particular branches of physics cultivated in Poland can, in the first approximation, be estimated on the basis of the number of physicists specializing in those branches. The relevant figures are summarized in Table 1 and relate to holders of the degree of master of science in physics, awarded after graduating from a $4.5-5$ year university course and submitting a thesis presenting an original scientific contribution.

As seen from the Table, solid state physics and nuclear physics are the fields most cultivated. The comparatively large number of theoretical physicists is also an interesting feature. On the other hand, applied physics is cultivated by a relatively small number of researchers, which causes deep concern on our part.

I shall now summarize the main lines of research conducted by Polish physicists excluding geophysics, biophysics and astronomy.

Table 1 - Workers with Higher Education Employed as Physicists

\begin{tabular}{|c|c|c|}
\hline Speciality & Number of workers & Percentage \\
\hline $\begin{array}{l}\text { Solid state physics } \\
\text { Nuclear physics } \\
\text { Applied physics } \\
\text { Biophysics } \\
\text { Optics } \\
\text { Acoustics } \\
\text { Geophysics } \\
\text { Other branches of physics }\end{array}$ & $\begin{array}{r}981 \\
470 \\
182 \\
167 \\
158 \\
123 \\
69 \\
469\end{array}$ & $\begin{array}{r}19.9 \\
9.5 \\
3.7 \\
3.4 \\
3.2 \\
2.5 \\
1.4 \\
9.5\end{array}$ \\
\hline \multicolumn{3}{|c|}{$\begin{array}{l}\text { Different branches of physics } \\
\text { specified in the questionnaires as : } \\
\text { Experimental physics } \\
\text { Theoretical physics } \\
\text { Not specified }\end{array}$} \\
\hline & Total & 100.0 \\
\hline
\end{tabular}

\section{Main Lines of Research}

Solid state physics is among the most rapidly developing branches of physics in Poland. Studies are conducted on a broad front and at extreme conditions - superhigh pressure, very high magnetic field and very low temperature, the most advanced being on semiconductors which have been going on since 1947. Several centres are concerned (Warsaw, Cracow, Wroclaw, Lublin) but the most intensive studies, both experimental and theoretical, are conducted in Warsaw. They concentrate on electron processes in semiconductor compounds and on the properties of new semiconductor materials. It seems that studies of the structure of energy bands in small gap semiconductors can be regarded as a Polish speciality.

Another important domain is magnetism. Initially it was approached chiefly theoretically, but later, an experimental programme was also started, that was partly connected with the launching of the Polish computer industry. Research on magnetism is conducted in several centres, but particularly in Poznań, Warsaw, Wroclaw and Cracow. In recent years, some activity is to be seen in the physics of metals (Katowice) which had been limited until then to the study of the crystalline structure of metals.

Investigations into the condensed phase go beyond the conventional limits of solid state physics. Much attention is paid to the study of the structure and dynamics of molecular liquids and liquid crystals, a field in which some pioneering work was performed in Cracow as early as 1924. Today such research is conducted in Cracow, Poznań and Warsaw.

Structural studies take place in Poland on a fairly wide scale, and are conducted chiefly in Wroclaw, Poz- nań, Cracow and Warsaw. These studies often go beyond the borders of physics and assume an interdisciplinary form, encompassing fundamental problems of chemistry, biology, crystallography, etc. The range of experimental methods used is also quite wide, from optical and X-ray spectroscopy, electron microscopy, electron paramagnetic resonance, which are used practically in every centre where structural investigations are conducted, to methods developed and used chiefly in Cracow and Warsaw, based on Mössbauer spectroscopy, slowneutron scattering, perturbed angular correlations and distributions of gamma-rays.

Closely related to structural studies are molecular physics and optics which are developing widely. Molecular physics is exercized practically in all Polish academic centres, but most intensely in Poznań, Wroclaw, Cracow, Gdańsk and Warsaw. These studies include investigations into the physics of dielectrics, including nonlinear dielectric effects, spectroscopy and radiospectroscopy (NMR and EPR under high pressures), molecular motions and interactions, ferroelectric substances.

Optical research has in Poland a long-standing tradition, especially as regards atomic and molecular optics, these branches constituting up to 1939 the chief sphere of Polish physics research. After World War II, this research was continued both experimentally and theoretically. Similarly, research in the domain of non-linear optics has been conducted in Poland for many years, even before the first laser was designed.

Today the main concern of Polish opticians is laser spectroscopy, and especially multiphoton processes (Warsaw, Poznań, Cracow, Toruń). In the sphere of non-linear optics, studies are conducted on self-focusing of light beams and on their scattering in complex molecular systems (Poz- 
nań, Warsaw). Experimental studies are also under way with the object of building new, highly efficient dye lasers and associated with this, theoretical work on the operating mechanism of such lasers (Warsaw, Toruń). For a decade now, research has been conducted in the field of holography.

Since 1955, plasma physics has been developed in Poland. In Warsaw, the main effort concentrates on thermonuclear fusion by ion implosion (using a cylindrical system of multirod electrodes), on the generation and heating of plasma within magnetic traps, and on plasma-focus experiments. Strong emphasis is also laid on the realization of nuclear microfusion by heating of the plasma in a laser-focus experiment; concentric chemical explosions have also been used for plasma compression.

Nuclear physics has a relatively long tradition in Poland and is one of the branches of physics most developed. As early as 1913, investigations were started in Warsaw on natural radioactivity under the patronage of Maria Sklodowska-Curie and in the early thirties, research turned to induced radioactivity. The first Polish particle accelerator was put into operation at the University of Warsaw in 1937.

Today, nuclear physics is cultivated on a wide scale in several centres, chiefly in Warsaw and Cracow, and to a smaller extent in Lublin, Lódź, Katowice and Wroclaw.

The investigations, experimental and theoretical, concentrate chiefly on the structure of nuclei and on nuclear reaction mechanisms. In structural problems, the attention of Warsaw physicists concentrates on nuclei from the $s-d$ and $f-p$ shells, from the regions of strong deformation and transitional nuclei. Much interest is focused on nuclei far from the stability line including the superheavy, and also nuclei in states with very high spin. In recent years also, investigations have been conducted on subcoulomb neutron transfer, aimed at the acquisition of information on neutron distribution in nuclei. Cracow physicists are particularly concerned with the optical model of complex particles interaction, cluster structure of light nuclei, and measurements of static and dynamic nuclear moments. Research on nuclear reaction mechanisms concerns mainly fast nucleon-pion and heavy ion-induced reactions (Warsaw and (racow).

Considerable difficulty is encountered in the further development of nuclear physics in Poland due to the lack of suitable, modern accelerators.
The Warsaw centre has at its disposal only one 3.5 MV van de Graaff generator, one $10 \mathrm{MeV}$ proton linac and one $30 \mathrm{MeV}$ betatron ; Cracow physicists have at their disposal a cyclotron which accelerates alpha-particles up to $28 \mathrm{MeV}$. In addition, in Warsaw, there are two research reactors, one of $10 \mathrm{MW}$ and the other of $30 \mathrm{MW}$. This very modest equipment does not satisfy the needs of the numerous staff, so a large part of the research programme is executed within international collaborations, based on the accelerators available in foreign laboratories. Significant assistance is obtained in this respect from the Joint Institute for Nuclear Research in Dubna, but also from some French, Swiss, Scandinavian, U.S. and other institutes. It is hoped that in the course of the next few years, the home situation will improve considerably, as the University of Warsaw has started building recently an isochronous cyclotron U $200(200 \mathrm{~cm}$ in diameter) which will accelerate heavy ions up to energies exceeding 10 $\mathrm{MeV} /$ nucleon.

High energy and elementary particle physics is also among the most advanced branches of physics in Poland, having been cultivated for 30 years. Studies of cosmic rays were started in Cracow directly after World War II and several years later research was undertaken in Warsaw and Cracow on high energy nuclear interactions, which brought in 1952 the discovery in the Warsaw laboratory of the first hypernucleus.

At present, the main lines of experimental and theoretical research in the field of high energy and elementary particle physics are, first of all, studies on multiparticle production in the hadron-nucleon and hadron-nucleus interaction, physics of hypernuclei, nucleus-nucleus interactions at relativistic energies, the theory of elementary particles, and lepton-nucleon interactions with production of hadrons. Furthemore, studies are in progress on extensive air showers of cosmic rays as well as interactions at supra-accelerator energies conducted in Lódź.

Even more than in nuclear physics, experimental research in the field of high energy and elementary particles is dependent on wide international collaboration. There are neither highenergy accelerators in this country nor any plans for building them in the future. However, a very good cooperation is established with the Joint Institute for Nuclear Research in Dubna and some Soviet centres of high energy physics (Serpukhov), as well as with the CERN and other WestEuropean and American laboratories.

Theoretical physics is also widely exercised and is continually popular with graduate physicists. An appreciable part of the research programmes executed by theoreticians is closely linked with experimental investigations and conducted in the same institutes. This applies in particular to nuclear physics, high energy and elementary particles physics, molecular physics and optics, branches already reviewed above.

In addition, theoretical investigations are conducted, first of all on classical and quantum field theory and in particular on the theory of gravitation and its applications to relativistic astrophysics (Warsaw, Wroclaw and Cracow) and on statistical physics with its applications, especially to the theory of condensed phase, notably in Wroclaw. Research is also conducted on the border line between statistical physics and the theory of information (Toruń).

Considerable attention is also concentrated on mathematical physics, which is exercized chiefly in Warsaw, Wroclaw and Toruń. Among the crucial problems here are the applications of differential geometry in physics.

The research potential of Polish physics, both as regards man-power and equipment, is expanding from year to year. New scientific institutions are being organized in which new research tasks are undertaken ; branches of the existing universities are set up to train new physicists for which there is a great demand in different sectors of the national economy, a demand which will almost double by 1990 . At present more than 600 physicists graduate annually from Polish universities and pedagogic institutes, the number of students starting physical education totalling about 1500. International collaboration is also systematically developing in the framework of both official bilateral agreements concluded between the respective governments and individual understandings between institutes and scientists.

International symposia and seminars on different physical problems are organized in Poland, some of which, as for example, the annual summer and winter schools in nuclear physics and theoretical physics, have gained international recognition and wide popularity among scientists.

In general, we can say that the development of physics in Poland is auspicious and we look hopefully to the future. 